\title{
Analytical Solution of Non-Isothermal Diffusion-Reaction Processes and Effectiveness Factors
}

\author{
Vembu Ananthaswamy and Lakshmanan Rajendran \\ Department of Mathematics, The Madura College, TamilNadu, Madurai 625011, India \\ Correspondence should be addressed to Lakshmanan Rajendran; raj_sms@rediffmail.com
}

Received 13 September 2012; Accepted 31 October 2012

Academic Editors: J. M. Farrar, E. B. Starikov, and A. Vergara

Copyright (C) 2013 V. Ananthaswamy and L. Rajendran. This is an open access article distributed under the Creative Commons Attribution License, which permits unrestricted use, distribution, and reproduction in any medium, provided the original work is properly cited.

\begin{abstract}
The mathematical modeling of nonlinear boundary value problems in catalytically chemical reactor is discussed. In this paper, we obtain the approximate analytical solution and the effectiveness factors for the evolution of single-step transformations under non-isothermal conditions using homotopy perturbation method. We have applied it to many reaction models and obtained very simple analytical expressions for the shape of the corresponding transformation rate peaks. These analytical solutions represent a significant simplification of the system's description allowing easy curve fitting to experiment. The accuracy achieved with our method is checked against several reaction models and numerical results. A satisfactory agreement is noted.
\end{abstract}

\section{Introduction}

Non-isothermal systems, where reaction and diffusion take place, are typical in the chemical process industry [1] and also in biological systems [2-4]. The chemical reaction is always central in these systems, because the rate of the reaction often will determine how fast chemicals can be produced. A high rate can be realized when the reaction is far from equilibrium. But an operation far from equilibrium is also an operation in which the energy dissipation is large. With the present interest to save valuable resources, chemical reactors should be studied also from the perspective of obtaining a more energy-efficient operation, in addition to maintaining the production of chemicals. In biological systems, one may expect that energy efficiency is an issue of survival, especially under harsh conditions [5]. In such cases and probably many others, a thermodynamic description will be important to understand the transport phenomena involved $[4,6]$. Studies of minimum energy dissipation start with an expression for the entropy production [7-9].

Chemical reactions are inherently non-linear processes, and are most successfully described in the field of reaction kinetics by the law of mass action $[10,11]$. The reaction rate is not commonly expressed as a function of the reaction
Gibbs energy. This is not surprising, because classical nonequilibrium thermodynamics $[12,13]$ assumes a linear relation between these two variables, and experimental evidence indicates that this is only correct very close to chemical equilibrium. The first to address this problem successfully was Kramers [14] who described the reaction as a diffusion process along a reaction coordinate.

The extension in the context of non-equilibrium thermodynamics was first proposed by Prigogine and Mazur [15-17]. By integrating over these variables to obtain the thermodynamic level, one can describe several phenomena, which are non-linear on the macroscopic level, and which retain a linear force-flux relation on the mesocopic level. This applies not only to chemical reactions [18], but for instance also to adsorption [19], nucleation [20], electrode over potentials [21] and active transport in biology [4]. The number of cases studied is now growing fast. The coupling of chemical reactions to other processes is then important $[8,18,22-$ 25]. Non-equilibrium thermodynamics is not only a theory for transport processes, it is also a theory for fluctuations. It has been demonstrated that the fluctuating contributions to the thermodynamic fluxes in a non-equilibrium system satisfy the fluctuation-dissipation theorem just like they do in equilibrium [26]. 
The theory of non-equilibrium thermodynamics is based on the assumption of local thermodynamic equilibrium. The validity of this assumption has been established by molecular dynamics simulations in several cases [27-29]. Fluctuations and the resulting correlation functions away from equilibrium were then not considered. One of the major findings has been that although local equilibrium is valid for the description of the mean values of thermodynamic fields, it is no longer valid for the description of the fluctuations around their average non-equilibrium values [26]. Recently Vergara et al. [30] developed the multicomponent diffusion system including cross-term diffusion coefficients relating to flux of the component $i$ to concentration gradients of component $j$. But in our problem the cross-diffusion is neglected. Ikeda et al. [31] analyzed this problem for a reaction-diffusion problem with a temperature gradient using a linear approximation for the description of the reaction. For the reaction-diffusion problem the assumption of local equilibrium has to be extended to be valid also along the reaction coordinate. However, to the best of our knowledge, till date no general analytical expressions of mass concentrations and effectiveness factors have been reported. The purpose of this communication is to derive the approximate analytical expression of mass concentration for planar particles by solving the non-linear differential equations using He's homotopy perturbation method [32-35].

\section{Mathematical Formulation of the Problem and Analysis}

The mathematical description of a catalytic chemical reactor $(m, q): m A+q B \rightarrow P$ is given by [1]

$$
\begin{gathered}
\frac{d^{2} u}{d x^{2}}-\phi^{2} f(u)=0 \quad \text { for } 0<x<L, \\
u=1 \quad \text { at } x=L, \quad \frac{d u}{d x}=0 \quad \text { at } x=0,
\end{gathered}
$$

where $x$ is the spatial coordinate, $f(u)$ is the reaction rate function (which is non-linear), and $L$ is the half thickness of porous slab $(m)$. The mass concentration $u$ is defined as the following function:

$$
u=\frac{C_{A}}{C_{\mathrm{AS}}},
$$

where $C_{A}$ is the volumetric molar concentration of the key component $A, C_{\mathrm{AS}}$ is the surface value of the key component $A$, and $\phi$ is the corresponding Thiele modulus which is defined as follows:

$$
\phi=\sqrt{\frac{L^{2} k_{S} C_{\mathrm{AS}}^{m-1} C_{\mathrm{BS}}^{p}}{D_{A}}} .
$$

Here $k_{S}, D_{A}$, and $C_{\mathrm{BS}}$ are the specific kinetic constant, effective diffusivity coefficient, and the dimensionless concentration of the component $B$, respectively. Also the temperature $T$ and the mass concentration $u$ are no longer independent, which satisfies the following relation:

$$
T=d(1-u)+1 \text {, }
$$

where $d$ is the thermicity of the reaction and is defined as follows:

$$
d=\frac{D_{A} C_{\mathrm{AS}}(-\Delta H)}{k T_{S}} .
$$

Here $-\Delta H$ is the reaction heat; $k$ is the effective thermal conductivity inside the porous slab; and $T_{S}$ is the dimensional temperature at the external pellet surface. $\Delta H / T_{S}$ represents the entropy change " $\Delta S$ " of a system under this process. Entropy increases in all spontaneous processes. Hence entropy may be regarded as a measure of disorder or randomness of the molecules of the system. For isothermal process, the entropy changes of the universe during a reversible process are zero. The entropy of the universe increases in an irreversible process. The parameter $d$ represents the deviation from isothermal conditions, being $d<0$ and $d>0$ for endothermic and exothermic reactions, respectively. Now the dimensionless reaction rate function $f(u)$ including $(1)$ is given by

$$
f(u)=u^{m+q} \exp \left[\frac{c(1-u)}{d(1-u)+1}\right],
$$

where the parameter $c$ is defined as follows:

$$
c=\gamma \cdot d
$$

where $\gamma$ is the Arrhenius group and is defined as

$$
\gamma=\frac{E}{R T_{S}} .
$$

Here $E$ denotes the activation energy; $R$ is the universal gas constant. Hence the corresponding non-linear boundary value problem is given by

$$
\begin{gathered}
\frac{d^{2} u}{d x^{2}}-\phi^{2} u^{m+q} \exp \left[\frac{c(1-u)}{d(1-u)+1}\right]=0 \quad \text { for } 0<x<L, \\
u=1 \quad \text { for } x=L, \quad \frac{d u}{d x}=0 \quad \text { for } x=0 .
\end{gathered}
$$

Using the following dimensionless variables:

$$
z=1-\frac{x}{L}, \quad y=1-u
$$

Now (9) becomes in dimensionless form as follows:

$$
\begin{gathered}
\frac{d^{2} y}{d z^{2}}-\phi^{2}(1-y)^{m+q} \exp \left[\frac{c y}{d y+1}\right]=0 \quad \text { for } 0<z<1 \\
y=0 \quad \text { for } z=0, \quad \frac{d y}{d z}=0 \quad \text { for } z=1
\end{gathered}
$$

The internal effectiveness factor $(\eta)$ is a measure of the relative importance of diffusion to reaction limitations. That is,

$$
\eta \equiv \frac{\text { actual overall reaction rate }}{\text { rate if entire surface were exposed to } C_{\mathrm{AS}}, T_{S}} .
$$


The effectiveness factor for the heterogeneous chemical reaction is [22-26]

$$
\eta=\frac{1}{\phi^{2}}\left[\frac{d y}{d z}\right]_{z=0}
$$

\section{Solution of Boundary Value Problem Using HPM}

Recently, many authors have applied the homotopy perturbation method (HPM) to solve the non-linear problems in physics and engineering sciences [36-39]. Recently this method is also used to solve some of the non-linear problem in physical sciences [32-34]. This method is a combination of homotopy in topology and classic perturbation techniques. He used to solve the Lighthill equation [32], the Diffusion equation [33], and the Blasius equation [34, 35]. The HPM is unique in its applicability, accuracy and efficiency. The HPM uses the imbedding parameter $p$ as a small parameter, and only a few iterations are needed to search for an asymptotic solution. Using this method, we can obtain the following solution to (11) and (12) for the following three cases (see Appendices A-C):

Case 1. When the reaction orders $q=0$ and $m=0$, the analytical solution of (11) to (12) using homotopy perturbation method $[35,40-44]$ is

$$
\begin{aligned}
y(z)= & {\left[\frac{d\left(3+\tan ^{2}(k)\right)}{2 c^{2}}-\frac{1}{c}\right]+\left[\frac{1}{c}+k_{1}\right] \cos (k z) } \\
& +\left[\frac{\tan (k)}{c}+k_{2}\right](\sin (k z)) \\
& -\left[\frac{\phi^{2} d\left(\left(1-\tan ^{2}(k)\right) \cos (2 k z)+2 \tan (k) \sin (2 k z)\right)}{6 c k^{2}}\right] \\
& +\left[\frac{\phi^{2} d(\tan (k) z \cos (k z)-z \sin (k z))}{k c}\right],
\end{aligned}
$$

where

$$
\begin{gathered}
k=\phi \sqrt{c}, \\
k_{1}=\left[\frac{\phi^{2} d\left(1-\tan ^{2}(k)\right)}{6 c k^{2}}\right]-\left[\frac{d\left(3+\tan ^{2}(k)\right)}{2 c^{2}}\right], \\
k_{2}=\frac{1}{k \cos (k)}\left\{\left[k k_{1} \sin (k)\right]-\left(\frac{\phi^{2} d}{3 c k}\right)\right. \\
\times\left[\left(1-\tan ^{2}(k)\right) \sin (2 k)\right. \\
\left.-2 \tan ^{2}(k) \cos (2 k)\right] \\
-\left(\frac{\phi^{2} d}{k c}\right)[\tan (k)(\cos (k)-k \sin (k)) \\
-(\sin (k)+k \cos (k))]\},
\end{gathered}
$$

provided $c \neq 0$. Using (14), the effectiveness factor $\eta$ is given by

$$
\begin{aligned}
\eta= & {\left[\frac{\sin (a \sqrt{c})}{a \sqrt{c} \cos (a \sqrt{c})}\right]-\left[\frac{d \sin (a \sqrt{c})}{a c^{3 / 2} \cos (a \sqrt{c})}\right] } \\
& -\left[\frac{2 d \sin (a \sqrt{c})}{3 a c^{3 / 2} \cos ^{3}(a \sqrt{c})}\right]+\left[\frac{d}{a \cos ^{2}(a \sqrt{c})}\right] .
\end{aligned}
$$

Case 2. When the reaction orders $q=0$ and $m=1$, we can obtain the analytical solution of (11) to (12) using homotopy perturbation method as follows:

$$
\begin{aligned}
y(z)= & {\left[\frac{(c+c d)\left(3+\tan ^{2}(a)\right)}{2(c-1)^{3}}-\frac{1}{(c-1)}\right] } \\
& +\left[\frac{1}{(c-1)}+a_{1}\right](\cos (a z))+\left[\frac{\tan (a)}{(c-1)}+a_{2}\right](\sin (a z)) \\
& -\left[\frac{\phi^{2}(c+c d)\left(\left(1-\tan ^{2}(a)\right) \cos (2 a z)+2 \tan (a) \sin (2 a z)\right)}{6 a^{2}(c-1)^{2}}\right] \\
& +\left[\frac{\phi^{2}(c+c d)(\tan (a) z \cos (a z)-z \sin (a z))}{a(c-1)^{2}}\right],
\end{aligned}
$$

where

$$
\begin{gathered}
a=\phi \sqrt{c-1}, \\
a_{1}=\left[\frac{\phi^{2}(c+c d)\left(1-\tan ^{2}(a)\right)}{6 a^{2}(c-1)^{2}}\right] \\
-\left[\frac{(c+c d)\left(3+\tan ^{2}(a)\right)}{2(c-1)^{3}}\right], \\
a_{2}=\frac{1}{a \cos (a)}\left\{\left(a a_{1} \sin (a)\right)-\left(\frac{\phi^{2}(c+c d)}{3 a(c-1)^{2}}\right)\right. \\
\times\left[\left(1-\tan ^{2}(a)\right) \sin (2 a)-2 \tan (a) \cos (2 a)\right] \\
+\left(\frac{\phi^{2}(c+c d)}{a(c-1)^{2}}\right)[\tan (a)(\cos (a)-a \sin (a)) \\
-(\sin (a)+a \cos (a))]\}
\end{gathered}
$$

provided $c \neq 1$. Using (14), the effectiveness factor $\eta$ is given by

$$
\begin{aligned}
\eta= & {\left[\frac{c^{2} \sin (a \sqrt{c-1})}{a \cos (a \sqrt{c-1})(c-1)^{5 / 2}}\right] } \\
& +\left[\frac{c d \sin (a \sqrt{c})}{(c-1)^{2} \cos ^{2}(a \sqrt{c-1})}\right] \\
& -\left[\frac{2 c d \sin (a \sqrt{c-1})}{3 a(c-1)^{5 / 2} \cos ^{3}(a \sqrt{c-1})}\right]
\end{aligned}
$$




$$
\begin{aligned}
& -\left[\frac{2 c \sin (a \sqrt{c-1})}{3 a \cos ^{3}(a \sqrt{c-1})(c-1)^{5 / 2}}\right] \\
& -\left[\frac{c d \sin (a \sqrt{c-1})}{3 a \cos (a \sqrt{c-1})(c-1)^{5 / 2}}\right] \\
& -\left[\frac{7 c \sin (a \sqrt{c-1})}{3 a \cos (a \sqrt{c-1})(c-1)^{5 / 2}}\right] \\
& +\left[\frac{c}{\cos ^{2}(a \sqrt{c-1})(c-1)^{2}}\right] \\
& +\left[\frac{\sin (a \sqrt{c-1})}{a \cos (a \sqrt{c-1})(c-1)^{5 / 2}}\right] .
\end{aligned}
$$

Case 3. When the reaction orders $q=1$ and $m=1$, the analytical solution of (11) to (12) using the homotopy perturbation method is given by

$$
\begin{aligned}
& y(z)=\left[\frac{(c d+2 c-1)\left(3+\tan ^{2}(b)\right)}{2(c-2)^{3}}-\frac{1}{(c-2)}\right] \\
& +\left[\frac{1}{(c-2)}+b_{1}\right](\cos (b z)) \\
& -\left[\frac{\phi^{2}(c d+2 c-1)\left(\left(1-\tan ^{2}(b)\right) \cos (2 b z)+2 \tan (b) \sin (2 b z)\right)}{6 b^{2}(c-2)^{2}}\right] \\
& +\left[\frac{\phi^{2}(c d+2 c-1)(\tan (b) z \cos (b z)-z \sin (b z))}{b(c-2)^{2}}\right] \\
& +\left[\frac{\tan (b)}{(c-2)}+b_{2}\right](\sin (b z)), \\
& b=\phi \sqrt{c-2}, \\
& b_{1}=\left[\frac{\phi^{2}(c d+2 c-1)\left(1-\tan ^{2}(b)\right)}{6 b^{2}(c-2)^{2}}\right] \\
& -\left[\frac{(c d+2 c-1)\left(3+\tan ^{2}(b)\right)}{2(c-2)^{3}}\right] \text {, } \\
& b_{2}=\left(\frac{1}{b \cos (b)}\right)\left\{b b_{1} \sin (b)-\left[\frac{\phi^{2}(c d+2 c-1)}{3 b(c-2)^{2}}\right]\right. \\
& \times\left(\left(1-\tan ^{2}(b)\right) \sin (2 b)\right. \\
& -2 \tan (b) \cos (2 b)) \\
& +\left[\frac{\phi^{2}(c d+2 c-1)}{b(c-2)^{2}}\right] \\
& \times(\tan (b)(\cos (b)-b \sin (b)) \\
& -(\sin (b)+b \cos (b)))\} \text {. }
\end{aligned}
$$

The above expression is valid only if $c \neq 2$. Using (14), the effectiveness factor $\eta$ is given by

$$
\begin{aligned}
& \eta=\left[\frac{c^{2} \sin (a \sqrt{c-2})}{a \cos (a \sqrt{c-2})(c-2)^{5 / 2}}\right] \\
& +\left[\frac{c d \sin (a \sqrt{c})}{(c-2)^{2} \cos ^{2}(a \sqrt{c-2})}\right] \\
& -\left[\frac{2 c d \sin (a \sqrt{c-2})}{3 a(c-2)^{5 / 2} \cos ^{3}(a \sqrt{c-2})}\right] \\
& -\left[\frac{4 c \sin (a \sqrt{c-2})}{3 a \cos ^{3}(a \sqrt{c-2})(c-2)^{5 / 2}}\right] \\
& -\left[\frac{c d \sin (a \sqrt{c-2})}{3 a \cos (a \sqrt{c-2})(c-2)^{5 / 2}}\right] \\
& -\left[\frac{14 c \sin (a \sqrt{c-2})}{3 a \cos (a \sqrt{c-2})(c-2)^{5 / 2}}\right] \\
& +\left[\frac{2 c}{\cos ^{2}(a \sqrt{c-2})(c-2)^{2}}\right] \\
& +\left[\frac{13 \sin (a \sqrt{c-2})}{3 a \cos (a \sqrt{c-1})(c-1)^{5 / 2}}\right] \\
& +\left[\frac{2 \sin (a \sqrt{c-2})}{3 a \cos (a \sqrt{c-2})(c-2)^{5 / 2}}\right] \\
& -\left[\frac{1}{\cos ^{2}(a \sqrt{c-2})(c-2)^{2}}\right] \text {. }
\end{aligned}
$$

\section{Numerical Simulation}

The non-linear equations (11) to (12) for the five cases are solved by numerical methods. The function pdex4, in Matlab software, is used to solve two-point boundary value problems (BVPs) for ordinary differential equations which are given in Appendices D-H. In Tables 1, 2, 3, 4, and 5, the numerical results are also compared with the obtained analytical expressions (see (15), (20), and (25)) and Villa et al. [1] results for some fixed value of $\phi=0.5$.

\section{Results and Discussions}

Tables 1-5 represent the dimensionless mass concentration $y(z)$ versus the dimensionless spatial coordinate $z$ for the following different values of the dimensionless parameters $c$ and $d$ :

(i) when $m=0, q=0, \phi=0.5, c>0$, and $d>0$.

(ii) when $m=0, q=0, \phi=0.5, c<0$, and $d<0$. 
TABLE 1: Comparison of our analytical expression of concentration profiles $y(z)$ with numerical results and Villa et al. [1] results corresponding to Case 1 , for $m=0, q=0$, and $\phi=0.5$, when $c>0$ and $d>0$.

\begin{tabular}{|c|c|c|c|c|c|c|c|c|}
\hline \multirow{2}{*}{$c$} & \multirow{2}{*}{$d$} & \multicolumn{7}{|c|}{ Dimensionless spatial coordinate $z$} \\
\hline & & & & 0.0 & 0.25 & 0.5 & 0.75 & 1.00 \\
\hline \multirow{3}{*}{2.5} & \multirow{3}{*}{0.5} & \multirow{3}{*}{$y(z)$} & Numerical & 0.000000 & 0.073340 & 0.128200 & 0.162200 & 0.173700 \\
\hline & & & Analytical (15) & 0.000000 & 0.070005 & 0.121817 & 0.153614 & 0.164329 \\
\hline & & & Villa et al. [1] & 0.000000 & 0.074132 & 0.129628 & 0.164013 & 0.175663 \\
\hline \multirow{3}{*}{2} & \multirow{3}{*}{0.4} & \multirow{3}{*}{$y(z)$} & Numerical & 0.000000 & 0.068130 & 0.118500 & 0.149500 & 0.160000 \\
\hline & & & Analytical (15) & 0.000000 & 0.066522 & 0.115447 & 0.145359 & 0.155421 \\
\hline & & & Villa et al. [1] & 0.000000 & 0.068637 & 0.119455 & 0.150699 & 0.161243 \\
\hline \multirow{3}{*}{1} & \multirow{3}{*}{0.2} & \multirow{3}{*}{$y(z)$} & Numerical & 0.000000 & 0.060340 & 0.104200 & 0.130700 & 0.139700 \\
\hline & & & Analytical (15) & 0.000000 & 0.060165 & 0.103798 & 0.130241 & 0.139099 \\
\hline & & & Villa et al. [1] & 0.000000 & 0.060524 & 0.104481 & 0.131152 & 0.140093 \\
\hline \multirow{3}{*}{0.5} & \multirow{3}{*}{0.1} & \multirow{3}{*}{$y(z)$} & Numerical & 0.000000 & 0.057310 & 0.098580 & 0.123500 & 0.131804 \\
\hline & & & Analytical (15) & 0.000000 & 0.057319 & 0.098578 & 0.123460 & 0.131775 \\
\hline & & & Villa et al. [1] & 0.000000 & 0.057394 & 0.098722 & 0.123652 & 0.131985 \\
\hline \multirow{3}{*}{0.25} & \multirow{3}{*}{0.05} & \multirow{3}{*}{$y(z)$} & Numerical & 0.000000 & 0.055960 & 0.096080 & 0.120200 & 0.128300 \\
\hline & & & Analytical (15) & 0.000000 & 0.055977 & 0.096116 & 0.120262 & 0.128321 \\
\hline & & & Villa et al. [1] & 0.000000 & 0.055994 & 0.096149 & 0.120306 & 0.128369 \\
\hline
\end{tabular}

TABLE 2: Comparison of our analytical expression of concentration profiles $y(z)$ with numerical results and Villa et al. [1] results corresponding to Case 1 , for $m=0, q=0$, and $\phi=0.5$, when $c<0$ and $d<0$.

\begin{tabular}{|c|c|c|c|c|c|c|c|c|}
\hline \multirow{2}{*}{$c$} & \multirow{2}{*}{$d$} & \multicolumn{7}{|c|}{ Dimensionless spatial coordinate $z$} \\
\hline & & & & 0.0 & 0.25 & 0.5 & 0.75 & 1.00 \\
\hline \multirow{3}{*}{-2.5} & \multirow{3}{*}{-0.5} & \multirow{3}{*}{$y(z)$} & Numerical & 0.000000 & 0.044356 & 0.074890 & 0.092878 & 0.098564 \\
\hline & & & Analytical (15) & 0.000000 & 0.044254 & 0.074608 & 0.092320 & 0.098140 \\
\hline & & & Villa et al. [1] & 0.000000 & 0.045077 & 0.076180 & 0.094425 & 0.100440 \\
\hline \multirow{3}{*}{-5} & \multirow{3}{*}{-0.5} & \multirow{3}{*}{$y(z)$} & Numerical & 0.000000 & 0.037821 & 0.062402 & 0.076523 & 0.080991 \\
\hline & & & Analytical (15) & 0.000000 & 0.037461 & 0.062200 & 0.076257 & 0.080814 \\
\hline & & & Villa et al. [1] & 0.000000 & 0.039354 & 0.065829 & 0.081121 & 0.086125 \\
\hline \multirow{3}{*}{-7.5} & \multirow{3}{*}{-0.5} & \multirow{3}{*}{$y(z)$} & Numerical & 0.000000 & 0.032841 & 0.053973 & 0.068000 & 0.070002 \\
\hline & & & Analytical (15) & 0.000000 & 0.032651 & 0.053454 & 0.067831 & 0.069662 \\
\hline & & & Villa et al. [1] & 0.000000 & 0.035385 & 0.058706 & 0.072018 & 0.076351 \\
\hline \multirow{3}{*}{-4} & \multirow{3}{*}{-0.2} & \multirow{3}{*}{$y(z)$} & Numerical & 0.000000 & 0.040345 & 0.067145 & 0.083100 & 0.087989 \\
\hline & & & Analytical (15) & 0.000000 & 0.040097 & 0.067028 & 0.082520 & 0.087574 \\
\hline & & & Villa et al. [1] & 0.000000 & 0.041559 & 0.069825 & 0.086265 & 0.091662 \\
\hline \multirow{3}{*}{-5} & \multirow{3}{*}{-0.2} & \multirow{3}{*}{$y(z)$} & Numerical & 0.000000 & 0.038110 & 0.062899 & 0.077121 & 0.081987 \\
\hline & & & Analytical (15) & 0.000000 & 0.037684 & 0.062628 & 0.076831 & 0.081440 \\
\hline & & & Villa et al. [1] & 0.000000 & 0.039551 & 0.066203 & 0.081619 & 0.086668 \\
\hline
\end{tabular}

(iii) when $m=1, q=0, \phi=0.5, c>0$, and $d>0$.

(iv) when $m=1, q=0, \phi=0.5, c<0$, and $d<0$.

(v) when $m=1, q=1, \phi=0.5, c>0$, and $d>0$.

From these tables it is evident that the values of the dimensionless mass concentration $y(z)$ decrease, when dimensionless parameters $c$ and $d$ decrease. In Tables 1-5, our analytical results for the mass concentrations $y(z)$ are compared with the numerical results and Villa et al. results [1]. Villa et al. [1] obtained the analytical solution of this problem only for taking the parametric restrictions.

In Tables 1-4, our analytical results are compared with the numerical results and Villa et al. [1] results. A good agreement between them is noted. In Table 5 for the Case 3, 
TABLE 3: Comparison of our analytical expression of concentration profiles $y(z)$ with numerical results and Villa et al. [1] results corresponding to Case 2, for $m=1, q=0$, and $\phi=0.5$, when $c>0$ and $d>0$.

\begin{tabular}{|c|c|c|c|c|c|c|c|c|}
\hline \multirow{2}{*}{$c$} & \multirow{2}{*}{$d$} & \multicolumn{7}{|c|}{ Dimensionless spatial coordinate $z$} \\
\hline & & & & 0.0 & 0.25 & 0.5 & 0.75 & 1.00 \\
\hline \multirow{3}{*}{2} & \multirow{3}{*}{0.4} & \multirow{3}{*}{$y(z)$} & Numerical & 0.000000 & 0.059510 & 0.102600 & 0.128600 & 0.137400 \\
\hline & & & Analytical (20) & 0.000000 & 0.058307 & 0.100271 & 0.125535 & 0.133966 \\
\hline & & & Villa et al. [1] & 0.000000 & 0.059654 & 0.102825 & 0.128938 & 0.137675 \\
\hline \multirow{3}{*}{1.01} & \multirow{3}{*}{0.2} & \multirow{3}{*}{$y(z)$} & Numerical & 0.000000 & 0.054380 & 0.093160 & 0.116400 & 0.124100 \\
\hline & & & Analytical (20) & 0.000000 & 0.055077 & 0.093182 & 0.116617 & 0.123772 \\
\hline & & & Villa et al. [1] $(c=1)$ & 0.000000 & 0.054316 & 0.093042 & 0.116241 & 0.123967 \\
\hline \multirow{3}{*}{0.5} & \multirow{3}{*}{0.1} & \multirow{3}{*}{$y(z)$} & Numerical & 0.000000 & 0.052160 & 0.089100 & 0.111100 & 0.118400 \\
\hline & & & Analytical (20) & 0.000000 & 0.052036 & 0.88869 & 0.110829 & 0.118125 \\
\hline & & & Villa et al. [1] & 0.000000 & 0.052090 & 0.088971 & 0.110966 & 0.118275 \\
\hline \multirow{3}{*}{0.25} & \multirow{3}{*}{0.05} & \multirow{3}{*}{$y(z)$} & Numerical & 0.000000 & 0.051160 & 0.087260 & 0.108700 & 0.115900 \\
\hline & & & Analytical (20) & 0.000000 & 0.051052 & 0.087075 & 0.108510 & 0.115625 \\
\hline & & & Villa et al. [1] & 0.000000 & 0.051065 & 0.087099 & 0.108542 & 0.115660 \\
\hline \multirow{3}{*}{0.1} & \multirow{3}{*}{0.02} & \multirow{3}{*}{$y(z)$} & Numerical & 0.000000 & 0.050590 & 0.086210 & 0.107400 & 0.114400 \\
\hline & & & Analytical (20) & 0.000000 & 0.050474 & 0.086019 & 0.107144 & 0.114151 \\
\hline & & & Villa et al. [1] & 0.000000 & 0.050476 & 0.086023 & 0.107149 & 0.114157 \\
\hline \multirow{3}{*}{0.01} & \multirow{3}{*}{0.002} & \multirow{3}{*}{$y(z)$} & Numerical & 0.000000 & 0.050250 & 0.085590 & 0.106600 & 0.113500 \\
\hline & & & Analytical (20) & 0.000000 & 0.050130 & 0.085393 & 0.106333 & 0.113278 \\
\hline & & & Villa et al. [1] & 0.000000 & 0.050130 & 0.085393 & 0.106333 & 0.113278 \\
\hline \multirow{3}{*}{0.001} & \multirow{3}{*}{0.0002} & \multirow{3}{*}{$y(z)$} & Numerical & 0.000000 & 0.050220 & 0.085530 & 0.106500 & 0.113500 \\
\hline & & & Analytical (20) & 0.000000 & 0.050096 & 0.085330 & 0.106253 & 0.113191 \\
\hline & & & Villa et al. [1] & 0.000000 & 0.050096 & 0.085330 & 0.106253 & 0.113191 \\
\hline
\end{tabular}

TABLE 4: Comparison of our analytical expression of concentration profiles $y(z)$ with numerical results and Villa et al. [1] results corresponding to Case 2, for $m=1, q=0$, and $\phi=0.5$, when $c<0$ and $d<0$.

\begin{tabular}{|c|c|c|c|c|c|c|c|c|}
\hline \multirow{2}{*}{$c$} & \multirow{2}{*}{$d$} & \multicolumn{7}{|c|}{ Dimensionless spatial coordinate $z$} \\
\hline & & & & 0.0 & 0.25 & 0.5 & 0.75 & 1.00 \\
\hline \multirow{3}{*}{-0.3} & \multirow{3}{*}{-0.1} & \multirow{3}{*}{$y(z)$} & Numerical & 0.000000 & 0.048975 & 0.083298 & 0.103610 & 0.110432 \\
\hline & & & Analytical (20) & 0.000000 & 0.048969 & 0.083271 & 0.103588 & 0.110316 \\
\hline & & & Villa et al. [1] & 0.000000 & 0.048985 & 0.083302 & 0.103629 & 0.110361 \\
\hline \multirow{3}{*}{-0.5} & \multirow{3}{*}{-0.1} & \multirow{3}{*}{$y(z)$} & Numerical & 0.000000 & 0.048943 & 0.082101 & 0.102105 & 0.108924 \\
\hline & & & Analytical (20) & 0.000000 & 0.048246 & 0.081952 & 0.101881 & 0.108475 \\
\hline & & & Villa et al. [1] & 0.000000 & 0.048288 & 0.082031 & 0.101987 & 0.108591 \\
\hline \multirow{3}{*}{-1.0} & \multirow{3}{*}{-0.1} & \multirow{3}{*}{$y(z)$} & Numerical & 0.000000 & 0.046786 & 0.078998 & 0.097997 & 0.104789 \\
\hline & & & Analytical (20) & 0.000000 & 0.046530 & 0.078818 & 0.097825 & 0.104101 \\
\hline & & & Villa et al. [1] & 0.000000 & 0.046673 & 0.079091 & 0.098191 & 0.104500 \\
\hline \multirow{3}{*}{-1.5} & \multirow{3}{*}{-0.1} & \multirow{3}{*}{$y(z)$} & Numerical & 0.000000 & 0.045001 & 0.076001 & 0.094255 & 0.100213 \\
\hline & & & Analytical (20) & 0.000000 & 0.044931 & 0.075901 & 0.094050 & 0.100030 \\
\hline & & & Villa et al. [1] & 0.000000 & 0.045214 & 0.076442 & 0.094775 & 0.100821 \\
\hline \multirow{3}{*}{-2.0} & \multirow{3}{*}{-0.1} & \multirow{3}{*}{$y(z)$} & Numerical & 0.000000 & 0.043678 & 0.073356 & 0.090611 & 0.096987 \\
\hline & & & Analytical (20) & 0.000000 & 0.043438 & 0.073179 & 0.090530 & 0.096234 \\
\hline & & & Villa et al. [1] & 0.000000 & 0.043888 & 0.074037 & 0.091678 & 0.097487 \\
\hline
\end{tabular}


TABLE 5: Comparison of our analytical expression of concentration profiles $y(z)$ with numerical results and Villa et al. [1] results corresponding to Case 3, for $m=1, q=1$, and $\phi=0.5$, when $c>0$ and $d>0$.

\begin{tabular}{|c|c|c|c|c|c|c|c|c|}
\hline \multirow{2}{*}{$c$} & \multirow{2}{*}{$d$} & \multicolumn{7}{|c|}{ Dimensionless spatial coordinate $z$} \\
\hline & & & & 0.0 & 0.25 & 0.5 & 0.75 & 1.00 \\
\hline \multirow{5}{*}{1.99} & \multirow{5}{*}{0.4} & \multirow{5}{*}{$y(z)$} & Numerical & 0.000000 & 0.053770 & 0.092021 & 0.114923 & 0.122500 \\
\hline & & & Analytical (25) & 0.000000 & 0.053168 & 0.090154 & 0.110179 & 0.119763 \\
\hline & & & (error \%) & $(0.0000)$ & $(1.1195)$ & $(2.0288)$ & $(4.0102)$ & $(2.2342)$ \\
\hline & & & Villa et al. [1] $(c=2)$ & 0.000000 & 0.013657 & 0.023410 & 0.029260 & 0.031210 \\
\hline & & & (error \%) & $(0.0000)$ & $(74.6010)$ & $(74.5601)$ & $(74.5394)$ & $(74.5224)$ \\
\hline \multirow{5}{*}{1.0} & \multirow{5}{*}{0.2} & \multirow{5}{*}{$y(z)$} & Numerical & 0.000000 & 0.049970 & 0.085070 & 0.105900 & 0.112800 \\
\hline & & & Analytical (25) & 0.000000 & 0.049630 & 0.084442 & 0.105065 & 0.108007 \\
\hline & & & (error \%) & $(0.0000)$ & $(0.6804)$ & $(0.7640)$ & $(0.7884)$ & $(4.2491)$ \\
\hline & & & Villa et al. [1] & 0.000000 & 0.013358 & 0.022862 & 0.028549 & 0.030442 \\
\hline & & & (error \%) & $(0.0000)$ & (73.2679) & $(73.1256)$ & $(73.0415)$ & $(73.0124)$ \\
\hline \multirow{5}{*}{0.5} & \multirow{5}{*}{0.1} & \multirow{5}{*}{$y(z)$} & Numerical & 0.000000 & 0.048300 & 0.082010 & 0.101900 & 0.108500 \\
\hline & & & Analytical (25) & 0.000000 & 0.048078 & 0.081632 & 0.101452 & 0.108007 \\
\hline & & & (error \%) & $(0.0000)$ & $(0.4596)$ & $(0.4609)$ & $(0.4396)$ & $(0.4543)$ \\
\hline & & & Villa et al. [1] & 0.000000 & 0.013216 & 0.022601 & 0.028210 & 0.030076 \\
\hline & & & (error \%) & $(0.0000)$ & $(72.6376)$ & $(72.7766)$ & $(72.3159)$ & $(72.2801)$ \\
\hline \multirow{5}{*}{0.25} & \multirow{5}{*}{0.05} & \multirow{5}{*}{$y(z)$} & Numerical & 0.000000 & 0.047510 & 0.080580 & 0.100100 & 0.106500 \\
\hline & & & Analytical (25) & 0.000000 & 0.046859 & 0.079420 & 0.098604 & 0.104941 \\
\hline & & & (error \%) & $(0.0000)$ & $(1.3702)$ & $(1.4395)$ & $(1.4945)$ & (1.4638) \\
\hline & & & Villa et al. [1] & 0.000000 & 0.013146 & 0.022473 & 0.028044 & 0.029897 \\
\hline & & & (error \%) & $(0.0000)$ & $(72.3300)$ & (72.1109) & $(71.9840)$ & $(71.9276)$ \\
\hline \multirow{5}{*}{0.1} & \multirow{5}{*}{0.02} & \multirow{5}{*}{$y(z)$} & Numerical & 0.000000 & 0.047060 & 0.079750 & 0.099010 & 0.105400 \\
\hline & & & Analytical (25) & 0.000000 & 0.046859 & 0.079420 & 0.098604 & 0.104941 \\
\hline & & & (error \%) & $(0.0000)$ & $(0.4271)$ & $(0.4137)$ & $(0.4100)$ & $(0.4354)$ \\
\hline & & & Villa et al. [1] & 0.000000 & 0.013104 & 0.022397 & 0.027946 & 0.029791 \\
\hline & & & (error \%) & $(0.0000)$ & $(72.1546)$ & (71.9159) & $(71.7745)$ & $(72.3531)$ \\
\hline \multirow{5}{*}{0.01} & \multirow{5}{*}{0.002} & \multirow{5}{*}{$y(z)$} & Numerical & 0.000000 & 0.046790 & 0.079260 & 0.098380 & 0.104700 \\
\hline & & & Analytical (25) & 0.000000 & 0.046588 & 0.078929 & 0.097971 & 0.104259 \\
\hline & & & (error \%) & $(0.0000)$ & $(0.4317)$ & $(0.41756)$ & $(0.4157)$ & $(0.4212)$ \\
\hline & & & Villa et al. [1] & 0.000000 & 0.013080 & 0.022352 & 0.027887 & 0.029727 \\
\hline & & & & $(0.0000)$ & $(72.0453)$ & $(71.7991)$ & $(71.6537)$ & (71.6074) \\
\hline \multirow{5}{*}{0.001} & \multirow{5}{*}{0.0002} & \multirow{5}{*}{$y(z)$} & Numerical & 0.000000 & 0.046760 & 0.079210 & 0.098320 & 0.104600 \\
\hline & & & Analytical (25) & 0.000000 & 0.046561 & 0.078880 & 0.097907 & 0.104191 \\
\hline & & & (error \%) & $(0.0000)$ & $(0.4255)$ & $(0.4166)$ & $(0.4200)$ & $(0.3910)$ \\
\hline & & & Villa et al. [1] & 0.000000 & 0.013077 & 0.022347 & 0.027881 & 0.029721 \\
\hline & & & (error \%) & $(0.0000)$ & (72.0337) & (71.7876) & $(71.6425)$ & $(71.5860)$ \\
\hline
\end{tabular}

our analytical results and Villa et al. [1] results are compared with the numerical results. Our analytical result gives good agreement with the numerical results. In Table 6, the effectiveness factors for the Cases 1 and 2, a satisfactory agreement between our results and Villa et al. [1] results is noted.

\section{Conclusion}

The steady state non-linear reaction-diffusion equation has been solved analytically and numerically. A simple and approximate dimensionless mass concentrations $y(z)$ are derived by using the HPM for all values of dimensionless parameters $c, d$, and $\phi$. The HPM is an extremely simple method and it is also a promising method to solve other nonlinear equations. This method can be easily extended to find the solution of all other non-linear equations. The proposed formulas are used to find the thiele module range, in which multiple values of the effectiveness factor should be searched. The present method is quick and efficient and is able to reduce 
TABle 6: Comparison of our effectiveness factors ((19), (24), and (29)), and Villa et al. [1] when $\phi=0.5$.

\begin{tabular}{|c|c|c|c|c|}
\hline \multirow{2}{*}{ Cases } & \multirow{2}{*}{$c$} & \multirow{2}{*}{$d$} & \multicolumn{2}{|c|}{ Effectiveness factors $(\eta)$} \\
\hline & & & Villa et al. [1] & Our results \\
\hline \multirow{10}{*}{ Case $1(m=0, q=0)$} & 2.5 & 0.5 & 1.3195 & 1.2526 \\
\hline & 2 & 0.4 & 1.2294 & 1.1952 \\
\hline & 1 & 0.2 & 1.0961 & 1.0903 \\
\hline & 0.5 & 0.1 & 1.0446 & 1.0434 \\
\hline & 0.25 & 0.05 & 1.0215 & 1.0213 \\
\hline & -2.5 & -0.5 & 0.8142 & 0.8280 \\
\hline & -5 & -0.5 & 0.7461 & 0.7157 \\
\hline & -7.5 & -0.5 & 0.6797 & 0.6360 \\
\hline & -4 & -0.2 & 0.7827 & 0.7592 \\
\hline & -5 & -0.2 & 0.7493 & 0.7193 \\
\hline \multirow{12}{*}{ Case $2(m=1, q=0)$} & 2.0 & 0.4 & 1.0820 & 1.0603 \\
\hline & 1.01 & 0.2 & $(c=1) 0.9940$ & 0.9890 \\
\hline & 0.5 & 0.1 & 0.9572 & 0.9564 \\
\hline & 0.25 & 0.05 & 0.9403 & 0.9401 \\
\hline & 0.1 & 0.02 & 0.9306 & 0.9305 \\
\hline & 0.01 & 0.002 & 0.9249 & 0.9249 \\
\hline & 0.001 & 0.0002 & 0.9243 & 0.9242 \\
\hline & -0.3 & -0.5 & 0.9059 & 0.9057 \\
\hline & -0.5 & -0.5 & 0.8944 & 0.8937 \\
\hline & -1.0 & -0.5 & 0.8676 & 0.8653 \\
\hline & -1.5 & -0.2 & 0.8435 & 0.8389 \\
\hline & -2.0 & -0.2 & 0.8214 & 0.8142 \\
\hline \multirow{7}{*}{ Case $3(m=1, q=1)$} & 1.99 & 0.4 & $(c=2) 0.9991$ & 0.9678 \\
\hline & 1.0 & 0.2 & 0.9793 & 0.9168 \\
\hline & 0.5 & 0.1 & 0.9699 & 0.8910 \\
\hline & 0.25 & 0.05 & 0.9653 & 0.8783 \\
\hline & 0.1 & 0.02 & 0.9626 & 0.8708 \\
\hline & 0.01 & 0.002 & 0.9609 & 0.8663 \\
\hline & 0.001 & 0.0002 & 0.9608 & 0.8658 \\
\hline
\end{tabular}

significantly the amount of computations in simulations of the catalytic chemical reactors.

\section{Appendices}

\section{A. Solution of Nonlinear Equations (11) and (12) Using HPM}

In this Appendix, we indicate how (15) in this paper is derived. To find the solution of (11) and (12), when $q=0$ and $m=0$. When $c y /(d y+1)$ small, then (11) reduces to

$$
\frac{d^{2} y}{d z^{2}}+\phi^{2}\left[1+c y-c d y^{2}\right]=0 \text {. }
$$

We construct the homotopy as follows:

$$
\begin{aligned}
& (1-p)\left[\frac{d^{2} y}{d z^{2}}+\phi^{2} c y+\phi^{2}\right] \\
& +p\left[\frac{d^{2} y}{d z^{2}}+\phi^{2} c y-\phi^{2} c d y^{2}+\phi^{2}\right]=0 .
\end{aligned}
$$

The analytical solution of (A.1) is 
Substituting (A.3) into (A.2), we get

$$
\begin{gathered}
(1-p)\left[\frac{d^{2}\left(y_{0}+p y_{1}+p^{2} y_{2}+\cdots\right)}{d z^{2}}\right. \\
+p\left[\frac{d^{2}\left(y_{0}+p y_{1}+p^{2} y_{2}+\cdots\right)}{d z^{2}}\right. \\
\left.+\phi^{2} c\left(y_{0}+p y_{1}+p^{2} y_{2}+\cdots\right)+\phi^{2}\right] \\
-\phi^{2} c d\left(y_{0}+p y_{1}+p^{2} y_{2}+\cdots\right) \\
\left.\left.+p^{2} y_{2}+\cdots\right)^{2}+\phi^{2}\right]=0 .
\end{gathered}
$$

Comparing the coefficients of like powers of $p$ in (A.4) we get

$$
\begin{gathered}
p^{0}: \frac{d^{2} y_{0}}{d z^{2}}+\phi^{2} c y_{0}+\phi^{2}=0, \\
p^{1}: \frac{d^{2} y_{1}}{d z^{2}}+\phi^{2} c y_{1}-\phi^{2} c d y_{0}^{2}=0 .
\end{gathered}
$$

The initial approximations are as follows:

$$
\begin{gathered}
y_{0}(0)=0, \quad y_{0}^{\prime}(1)=0, \\
y_{i}(0)=y_{i}^{\prime}(1)=0, \quad i=1,2,3 \ldots .
\end{gathered}
$$

Solving (A.5) and using the boundary conditions (A.6) we obtain the following results:

$$
\begin{aligned}
y_{0}= & \frac{1}{c}[\cos (k z)+\tan (k) \sin (k z)-1] \\
y_{1}= & k_{1} \cos (k z)+k_{2} \sin (k z)+\left[\frac{d\left(3+\tan ^{2}(k)\right)}{2 c^{2}}\right] \\
& -\left[\frac{\phi^{2} d\left(1-\tan ^{2}(k)\right) \cos (2 k z)}{6 c k^{2}}\right] \\
& -\left[\frac{\phi^{2} d \tan (k) \sin (2 k z)}{3 c k^{2}}\right] \\
& +\left[\frac{\phi^{2} d \tan (k) z \cos (k z)}{c k}\right]-\left[\frac{\phi^{2} d z \sin (k z)}{c k}\right],
\end{aligned}
$$

where $k, k_{1}$, and $k_{2}$ are defined in the text (16), (17), and (18), respectively.

According to the HPM, we can conclude that

$$
y=\lim _{p \rightarrow 1} y(t)=y_{0}+y_{1} .
$$

After putting (A.7) into (A.8) we obtain the solution in the text (15).

\section{B. Solution of Nonlinear Equations (11) and (12) Using HPM}

In this Appendix, we indicate how (20) in this paper is derived. To find the solution of (11) and (12), when $q=0$ and $m=1$. When $c y /(d y+1)$ small, then $(11)$ reduces to

$$
\frac{d^{2} y}{d z^{2}}+\phi^{2}\left[1+(c-1) y-(c+c d) y^{2}\right]=0
$$

We construct the homotopy as follows:

$$
\begin{aligned}
& (1-p)\left[\frac{d^{2} y}{d z^{2}}+\phi^{2}(c-1) y+\phi^{2}\right] \\
& +p\left[\frac{d^{2} y}{d z^{2}}+\phi^{2}(c-1) y-\phi^{2}(c+c d) y^{2}+\phi^{2}\right]=0 .
\end{aligned}
$$

The analytical solution of (B.1) is

$$
y=y_{0}+p y_{1}+p^{2} y_{2}+\cdots
$$

Substituting (B.3) into (B.2), we get

$$
\begin{aligned}
(1-p)\left[\frac{d^{2}\left(y_{0}+p y_{1}+p^{2} y_{2}+\cdots\right)}{d z^{2}}\right. \\
\left.+\phi^{2}(c-1)\left(y_{0}+p y_{1}+p^{2} y_{2}+\cdots\right)+\phi^{2}\right] \\
+p\left[\frac{d^{2}\left(y_{0}+p y_{1}+p^{2} y_{2}+\cdots\right)}{d z^{2}}+\phi^{2}(c-1)\right. \\
\quad \times\left(y_{0}+p y_{1}+p^{2} y_{2}+\cdots\right)-\phi^{2}(c+c d) \\
\left.\quad \times\left(y_{0}+p y_{1}+p^{2} y_{2}+\cdots\right)^{2}+\phi^{2}\right]=0 .
\end{aligned}
$$

Comparing the coefficients of like powers of $p$ in (B.4) we get

$$
\begin{gathered}
p^{0}: \frac{d^{2} y_{0}}{d z^{2}}+\phi^{2}(c-1) y_{0}+\phi^{2}=0, \\
p^{1}: \frac{d^{2} y_{1}}{d z^{2}}+\phi^{2}(c-1) y_{1}+\phi^{2}(c+c d) y_{0}^{2}=0 .
\end{gathered}
$$

The initial approximations are as follows:

$$
\begin{gathered}
y_{0}(0)=0, \quad y_{0}^{\prime}(1)=0, \\
y_{i}(0)=y_{i}^{\prime}(1)=0, \quad i=1,2,3 \ldots
\end{gathered}
$$


Solving (B.5) and using the boundary conditions (B.6) we obtain the following results:

$$
\begin{aligned}
y_{0} & =\left(\frac{1}{(c-1)}\right)[\cos (a z)+\tan (a) \sin (a z)-1], \\
y_{1}= & {\left[a_{1} \cos (a z)+a_{2} \sin (a z)\right]+\left[\frac{(c+c d)\left(3+\tan ^{2}(a)\right)}{2(c-1)^{3}}\right] } \\
& -\left[\frac{\phi^{2}(c+c d)\left(1-\tan ^{2}(a)\right) \cos (2 a z)}{6(c-1)^{2} a^{2}}\right] \\
- & {\left[\frac{\phi^{2}(c+c d) \tan (a) \sin (2 a z)}{3(c-1)^{2} a^{2}}\right] } \\
+ & {\left[\frac{\phi^{2}(c+c d) \tan (a) z \cos (a z)}{(c-1)^{2} a}\right] } \\
- & {\left[\frac{\phi^{2}(c+c d) z \sin (a z)}{(c-1)^{2} a}\right] }
\end{aligned}
$$

where $a, a_{1}$, and $a_{2}$ are defined in the text (21), (22), and (23) respectively.

According to the HPM, we can conclude that

$$
y=\lim _{p \rightarrow 1} y(t)=y_{0}+y_{1} .
$$

After putting (B.7) into (B.8) we obtain the solution in the text (20).

\section{Solution of Nonlinear Equations (11) and (12) Using HPM}

In this Appendix, we indicate how (25) in this paper is derived. To find the solution of (11) and (12), when $q=1$ and $m=1$.

When $c y /(d y+1)$ small, then (11) reduces to

$$
\frac{d^{2} y}{d z^{2}}+\phi^{2}\left[1+(c-2) y-(c d+2 c-1) y^{2}\right]=0 \text {. }
$$

We construct the homotopy as follows:

$$
\begin{aligned}
& (1-p)\left[\frac{d^{2} y}{d z^{2}}+\phi^{2}(c-2) y+\phi^{2}\right] \\
& +p\left[\frac{d^{2} y}{d z^{2}}+\phi^{2}(c-2) y-\phi^{2}(c d+2 c-1) y^{2}+\phi^{2}\right]=0 .
\end{aligned}
$$

The analytical solution of (C.1) is

$$
y=y_{0}+p y_{1}+p^{2} y_{2}+\cdots
$$

Substituting (C.3) into (C.2), we get

$$
\begin{aligned}
(1-p)\left[\frac{d^{2}\left(y_{0}+p y_{1}+p^{2} y_{2}+\cdots\right)}{d z^{2}}\right. \\
\left.+\phi^{2}(c-2)\left(y_{0}+p y_{1}+p^{2} y_{2}+\cdots\right)+\phi^{2}\right] \\
+p\left[\frac{d^{2}\left(y_{0}+p y_{1}+p^{2} y_{2}+\cdots\right)}{d z^{2}}+\phi^{2}(c-2)\right. \\
\quad \times\left(y_{0}+p y_{1}+p^{2} y_{2}+\cdots\right)-\phi^{2}(c d+2 c-1) \\
\left.\quad \times\left(y_{0}+p y_{1}+p^{2} y_{2}+\cdots\right)^{2}+\phi^{2}\right]=0 .
\end{aligned}
$$

Comparing the coefficients of like powers of $p$ in (C.4) we get

$$
\begin{gathered}
p^{0}: \frac{d^{2} y_{0}}{d z^{2}}+\phi^{2}(c-2) y_{0}+\phi^{2}=0, \\
p^{1}: \frac{d^{2} y_{1}}{d z^{2}}+\phi^{2}(c-2) y_{1}-\phi^{2}(c d+2 c-1) y_{0}^{2}=0 .
\end{gathered}
$$

The initial approximations is as follows:

$$
\begin{gathered}
y_{0}(0)=0, \quad y_{0}^{\prime}(1)=0, \\
y_{i}(0)=y_{i}^{\prime}(1)=0, \quad i=1,2,3 \ldots .
\end{gathered}
$$

Solving (C.5) and using the boundary conditions (C.6) we obtain the following results:

$$
\begin{aligned}
y_{0}= & \frac{1}{(c-2)}[\cos (b z)+\tan (b) \sin (b z)-1], \\
y_{1}= & {\left[b_{1} \cos (b z)+b_{2} \sin (b z)\right] } \\
& +\left[\frac{\left.(c d+2 c-1)\left(3+\tan ^{2}(b)\right)\right]}{2(c-2)^{3}}\right] \\
& -\left[\frac{\phi^{2}(c d+2 c-1)\left(1-\tan ^{2}(b)\right) \cos (2 b z)}{6(c-2)^{2} b^{2}}\right] \\
& -\left[\frac{\phi^{2}(c d+2 c-1) \tan (b) \sin (2 b z)}{3(c-2)^{2} b^{2}}\right] \\
& +\left[\frac{\phi^{2}(c d+2 c-1) \tan (b) z \cos (b z)}{(c-2)^{2} b}\right] \\
& -\left[\frac{\phi^{2}(c d+2 c-1) z \sin (b z)}{(c-2)^{2} b}\right],
\end{aligned}
$$

where $b, b_{1}$, and $b_{2}$ are defined in the text (26), (27), and (28), respectively.

According to the HPM, we can conclude that

$$
y=\lim _{p \rightarrow 1} y(t)=y_{0}+y_{1} .
$$


After putting (C.7) into (C.8) we obtain the solution in the text (25).

\section{MATLAB Program to Find the Numerical} Solution of Nonlinear Equations (11) and (12)

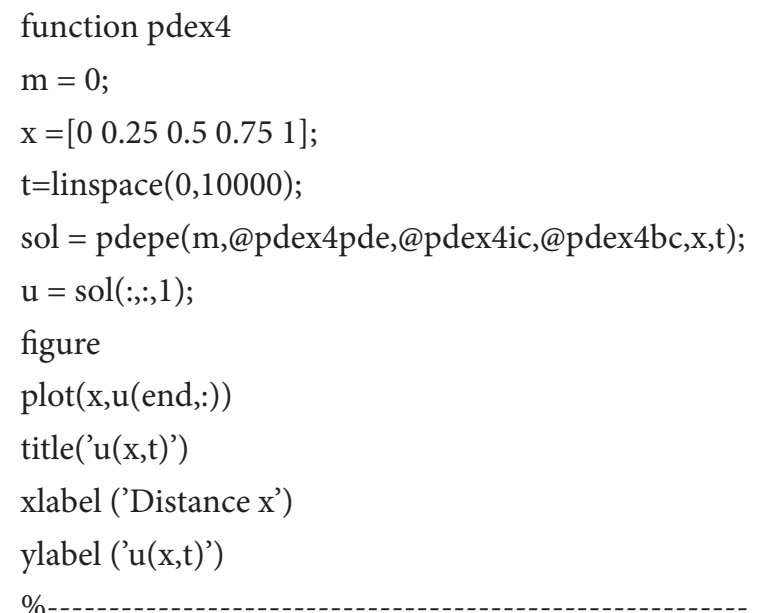

function $[c, f, s]=\operatorname{pdex} 4 p d e(x, t, u, D u D x)$

$c=1$;

$\mathrm{f}=\mathrm{DuDx}$

$\mathrm{a}=0.5$;

$\mathrm{c}=1$;

$\mathrm{d}=0.2$;

$\mathrm{m}=0$;

$\mathrm{p}=0$;

$\mathrm{F}=\mathrm{a}^{\wedge} 2^{*}(1-\mathrm{u})^{\wedge}(\mathrm{m}+\mathrm{p})^{*} \exp \left(\mathrm{c}^{*} \mathrm{u} /\left(1+\mathrm{d}^{*} \mathrm{u}\right)\right)$

$\mathrm{s}=\mathrm{F}$;

$\%$

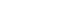

function $\mathrm{u} 0=\operatorname{pdex} 4 \mathrm{ic}(\mathrm{x}) ; \%$ create initial conditions $\mathrm{u} 0=1 ;$

$\%$

function[pl,ql,pr,qr] =pdex $4 b c(x l, u l, x r, u r, t) \quad \%$ create boundary conditions

$\mathrm{pl}=\mathrm{ul}$;

$\mathrm{ql}=0$;

pr $=0$;

$\mathrm{qr}=1$.

\section{E. MATLAB Program to Find the Numerical} Solution of Nonlinear Equations (11) and (12)

function pdex 4

$\mathrm{m}=0$;

$\mathrm{x}=\left[\begin{array}{lllll}0 & 0.25 & 0.5 & 0.75 & 1\end{array}\right]$;

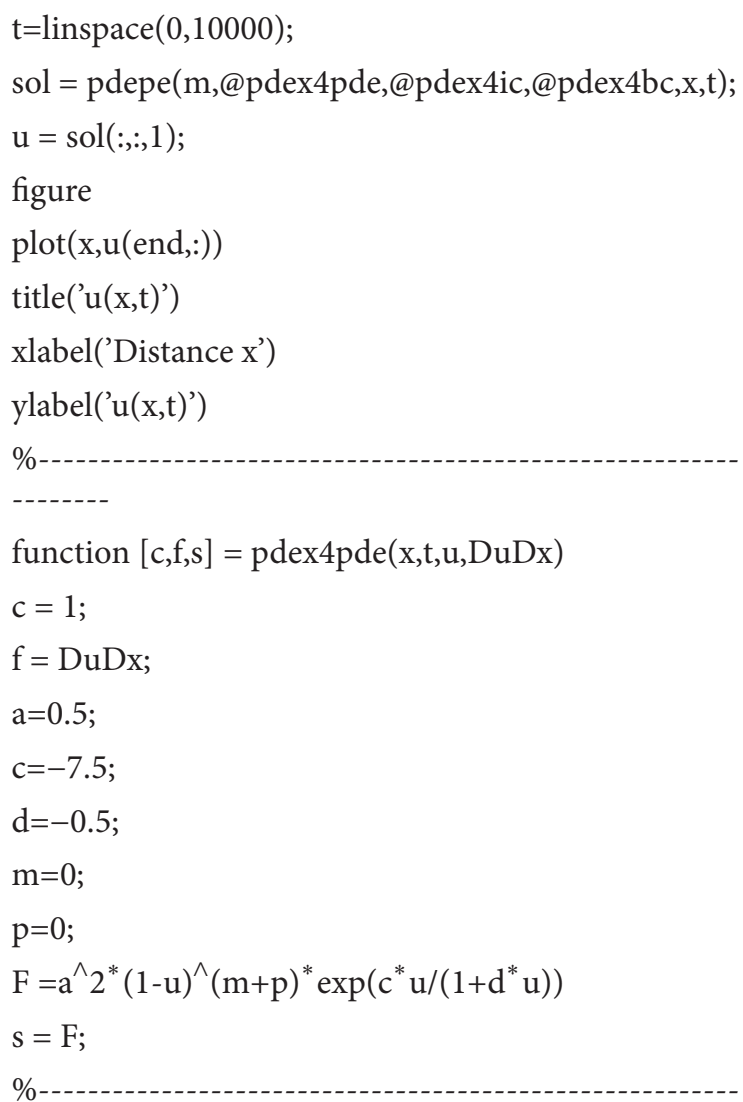

function $\mathrm{u} 0=\operatorname{pdex} 4 \mathrm{ic}(\mathrm{x}) ; \%$ create initial conditions $\mathrm{u} 0=1$;

$\%$

function [pl,ql,pr,qr]=pdex $4 \mathrm{bc}(\mathrm{xl}, \mathrm{ul}, \mathrm{xr}, \mathrm{ur}, \mathrm{t}) \quad \%$ create boundary conditions

$\mathrm{pl}=\mathrm{ul}$;

$\mathrm{ql}=0$;

$\mathrm{pr}=0$;

$\mathrm{qr}=1$.

\section{F. MATLAB Program to Find} the Numerical Solution of Non-Linear Equations (11) and (12)

function pdex 4

$\mathrm{m}=0$;

$\mathrm{X}=\left[\begin{array}{lllll}0 & 0.25 & 0.5 & 0.75 & 1\end{array}\right]$;

$\mathrm{t}=$ linspace $(0,10000)$;

sol=pdepe(m,@pdex4pde,@pdex4ic,@pdex4bc,x,t); $\mathrm{u}=\operatorname{sol}(:,:, 1)$;

figure

$\operatorname{plot}(\mathrm{x}, \mathrm{u}(\mathrm{end}, \mathrm{)}))$

title('u(x,t)') 


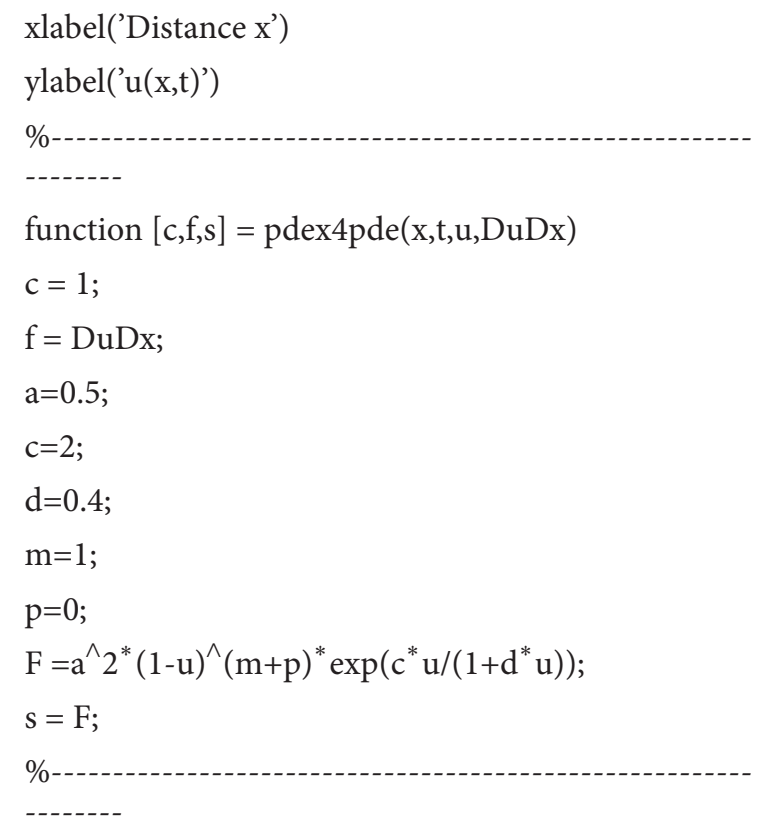

function $\mathrm{u} 0=\operatorname{pdex} 4 \mathrm{ic}(\mathrm{x}) ; \%$ create initial conditions $\mathrm{u} 0=1$;

$\%$

function[pl,ql,pr,qr] =pdex $4 \mathrm{bc}(\mathrm{xl}, \mathrm{ul}, \mathrm{xr}, \mathrm{ur}, \mathrm{t}) \quad \%$ create boundary conditions

$\mathrm{pl}=\mathrm{ul}$;

$\mathrm{ql}=0$;

pr $=0$;

$\mathrm{qr}=1$.

\section{G. MATLAB Program to Find} the Numerical Solution of Non-Linear Equations (11) and (12)

function pdex 4

$\mathrm{m}=0$;

$\mathrm{x}=\left[\begin{array}{lllll}0 & 0.25 & 0.5 & 0.75 & 1\end{array}\right]$;

$\mathrm{t}=$ linspace $(0,10000)$;

sol=pdepe $(\mathrm{m}, @ p d e x 4 p d e, @ p d e x 4 i c, @ p d e x 4 b c, x, t)$;

$\mathrm{u}=\operatorname{sol}(:,:, 1)$;

figure

$\operatorname{plot}(\mathrm{x}, \mathrm{u}(\mathrm{end}, \mathrm{)}))$

title('u(x,t)')

xlabel('Distance x')

ylabel('u(x,t)')

$\%$

--------

function $[\mathrm{c}, \mathrm{f}, \mathrm{s}]=\operatorname{pdex} 4 \mathrm{pde}(\mathrm{x}, \mathrm{t}, \mathrm{u}, \mathrm{DuDx})$

$c=1$

$$
\begin{aligned}
& \mathrm{f}=\mathrm{DuDx} \\
& \mathrm{a}=0.5 ; \\
& \mathrm{c}=-1.5 ; \\
& \mathrm{d}=-0.1 ; \\
& \mathrm{m}=1 ; \\
& \mathrm{p}=0 ; \\
& \mathrm{F}=\mathrm{a}^{\wedge} 2^{*}(1-\mathrm{u})^{\wedge}(\mathrm{m}+\mathrm{p})^{*} \exp \left(\mathrm{c}^{*} \mathrm{u} /\left(1+\mathrm{d}^{*} \mathrm{u}\right)\right) \\
& \mathrm{s}=\mathrm{F} ;
\end{aligned}
$$

function $[\mathrm{pl}, \mathrm{ql}, \mathrm{pr}, \mathrm{qr}]=\mathrm{pdex} 4 \mathrm{bc}(\mathrm{xl}, \mathrm{ul}, \mathrm{xr}, \mathrm{ur}, \mathrm{t}) \quad \%$ create boundary conditions

$$
\begin{aligned}
& \mathrm{pl}=\mathrm{ul} ; \\
& \mathrm{ql}=0 ; \\
& \mathrm{pr}=0 ; \\
& \mathrm{qr}=1
\end{aligned}
$$

\section{H. MATLAB Program to Find} the Numerical Solution of Non-Linear Equations (11) and (12)

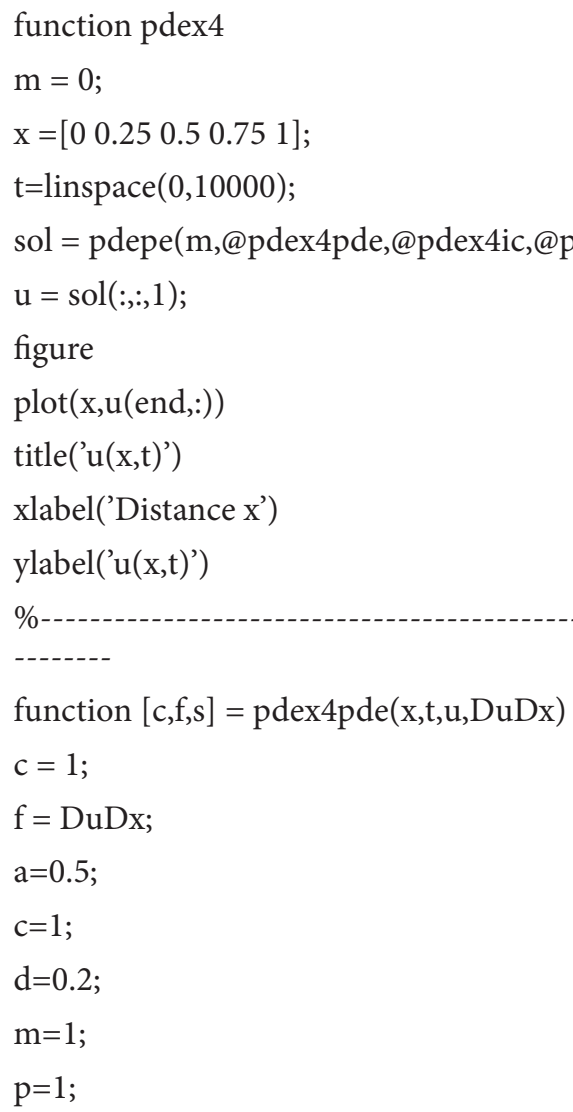




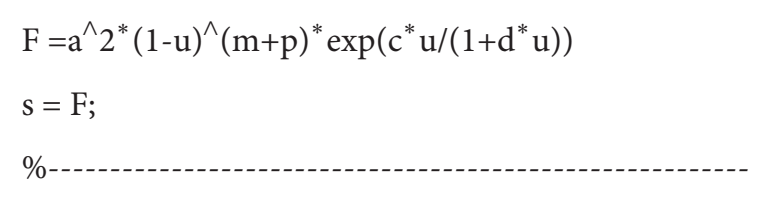

function $\mathrm{u} 0=\operatorname{pdex} 4 \mathrm{ic}(\mathrm{x}) ; \%$ create initial conditions

$\mathrm{u} 0=1$

$\%$

function[pl,ql,pr,qr]=pdex $4 \mathrm{bc}(\mathrm{xl}, \mathrm{ul}, \mathrm{xr}, \mathrm{ur}, \mathrm{t}) \quad \%$ create boundary conditions

$\mathrm{pl}=\mathrm{ul}$;

$\mathrm{ql}=0$;

$\mathrm{pr}=0$;

$\mathrm{qr}=1$.

\section{Nomenclature}

$\begin{array}{ll}x: & \text { Spatial coordinate }(m) \\ z: & \text { Dimensionless spatial coordinate } \\ u, y: & \text { Dimensionless mass concentration of } \\ f(u): & \text { reactive component } A \\ v, w: & \text { Dimensionless reaction rate } \\ L: & \text { Himensionless functions } \\ m, q: & \text { Whole numbers which denote the reaction } \\ P: & \text { order } \\ R: & \text { Product chemical component in a chemical } \\ T: & \text { Universal gas constant } \\ & \text { Functions which represent temperature } \\ C_{A}: & \left.\text { profile in the porous slab }{ }^{\circ} \mathrm{K}\right) \\ & \text { Volumetric molar concentration of the key } \\ C_{\mathrm{AS}}: & \text { Component } A \\ D_{A}: & \text { Surface value of the key component } A \\ k_{S} & \text { Effective diffusivity coefficient } \\ C_{\mathrm{BS}}: & \text { Specific kinetic constant } \\ & \text { Dimensionless concentration of component } \\ d: & \text { B Thermicity of the reaction } \\ \gamma: & \text { Arrhenius group } \\ c=\gamma \cdot d: & \text { Dimensionless parameter } \\ \phi: & \text { Thiele modulus } \\ \eta: & \text { Effectiveness factor for the heterogeneous } \\ & \text { chemical reaction. }\end{array}$

\section{Acknowledgments}

This work was supported by the Council of Scientific and Industrial Research (CSIR), no. 01 (2442)/10/EMR-II, Government of India. The authors are thankful to the Secretary, the Principal, The Madura College, Madurai, TamilNadu, India for their constant encouragement. The authors are very grateful to referees for their valuable suggestions.

\section{References}

[1] L. T. Villa, N. A. Acosta, and C. M. Albarracin, "A note on nonisothermal diffusion-reaction processes," International Journal of Pure and Applied Mathematics, vol. 71, no. 4, pp. 525-538, 2011.

[2] D. Bedeaux, I. Pagonabarraga, J. M. Ortiz De Zárate, J. V. Sengers, and S. Kjelstrup, "Mesoscopic non-equilibrium thermodynamics of non-isothermal reaction-diffusion," Physical Chemistry Chemical Physics, vol. 12, no. 39, pp. 12780-12793, 2010.

[3] G. F. Froment and K. B. Bischoff, Chemical Reactor Analysis and Design, John Wiley \& Sons, New York, NY, USA, 2nd edition, 1990.

[4] S. Kjelstrup, J. M. Rubi, and D. Bedeaux, "Energy dissipation in slipping biological pumps," Physical Chemistry Chemical Physics, vol. 7, no. 23, pp. 4009-4018, 2005.

[5] A. S. Blix, Arctic Animals, Tapir Academic, Trondheim, Norway, 2001.

[6] D. Bedeaux and S. Kjelstrup, "The measurable heat flux that accompanies active transport by $\mathrm{Ca}^{2+}$-ATPase," Physical Chemistry Chemical Physics, vol. 10, no. 48, pp. 7304-7317, 2008.

[7] E. Johannessen and S. Kjelstrup, "Numerical evidence for a "highway in state space" for reactors with minimum entropy production," Chemical Engineering Science, vol. 60, no. 5, pp. 1491-1495, 2005.

[8] S. Kjelstrup, D. Bedeaux, and E. Johannessen, Elements of Irreversible Thermodynamics for Engineers, Tapir Academic, Trondheim, Norway, 2007.

[9] L. V. Van Der Ham, J. Gross, A. Verkooijen, and S. Kjelstrup, "Efficient conversion of thermal energy into hydrogen: comparing two methods to reduce exergy losses in a sulfuric acid decomposition reactor," Industrial and Engineering Chemistry Research, vol. 48, no. 18, pp. 8500-8507, 2009.

[10] C. M. Guldberg and P. Waage, Forhandlinger: VIdenskabsSelskabet i ChrIstIanIa, 1864.

[11] H. Eyring and E. Eyring, Modern Chemical Kinetics, Chapman \& Hall, London, UK, 1965.

[12] L. Onsager, "Reciprocal relations in irreversible processes. I," Physical Review, vol. 37, no. 4, pp. 405-426, 1931.

[13] L. Onsager, "Reciprocal relations in irreversible processes. II," Physical Review, vol. 38, no. 12, pp. 2265-2279, 1931.

[14] H. A. Kramers, "Brownian motion in a field of force and the diffusion model of chemical reactions," Physica, vol. 7, no. 4, pp. 284-304, 1940.

[15] I. Prigogine and P. Mazur, "Sur l'extension de la thermodynamique aux phénomènes irreversibles liés aux degrés de liberté internes," Physica, vol. 19, no. 1-12, pp. 241-254, 1953.

[16] S. R. de Groot and P. Mazur, Non-Equilibrium Thermodynamics, North-Holland, Amsterdam, The Netherlands, 1962.

[17] D. Reguera, J. M. Rubí, and J. M. G. Vilar, "The mesoscopic dynamics of thermodynamic systems," Journal of Physical Chemistry B, vol. 109, no. 46, pp. 21502-21515, 2005.

[18] I. Pagonabarraga, A. Pérez-Madrid, and J. M. Rubí, "Fluctuating hydrodynamics approach to chemical reactions," Physica A, vol. 237, no. 1-2, pp. 205-219, 1997.

[19] I. Pagonabarraga and J. M. Rubí, "Derivation of the Langmuir adsorption equation from non-equilibrium thermodynamics," Physica A, vol. 188, no. 4, pp. 553-567, 1992.

[20] D. Reguera and J. M. Rubí, "Nonequilibrium translationalrotational effects in nucleation," Journal of Chemical Physics, vol. 115 , no. 15, pp. 7100-7106, 2001. 
[21] J. M. Rubi and S. Kjelstrup, "Mesoscopic nonequilibrium thermodynamics gives the same thermodynamic basis to ButlerVolmer and Nerst equations," Journal of Physical Chemistry B, vol. 107, no. 48, pp. 13471-13477, 2003.

[22] Y. Demirel and S. I. Sandler, "Effects of concentration and temperature on the coupled heat and mass transport in liquid mixtures," International Journal of Heat and Mass Transfer, vol. 45, no. 1, pp. 75-86, 2001.

[23] S. Kjelstrup and D. Bedeaux, "Elements of irreversible thermodynamics for engineers," in Applied Thermodynamics, A. H. Goodwin, J. V. Sengers, and C. J. Peters, Eds., IUPAC Chemical Data Series no. XX, chapter 14, Royal Society of Chemistry, London, UK, 2010.

[24] Y. Demirel, "Non-isothermal reaction-diffusion systems with thermodynamically coupled heat and mass transfer," Chemical Engineering Science, vol. 61, no. 10, pp. 3379-3385, 2006.

[25] Y. Demirel, "Modeling of thermodynamically coupled reactiontransport systems," Chemical Engineering Journal, vol. 139, no. 1, pp. 106-117, 2008.

[26] J. M. Ortiz de Zárate and J. V. Sengers, Hydrodynamic Fluctuations in Fluids and Fluid Mixtures, Elsevier, Amsterdam, The Netherlands, 2006.

[27] B. Hafskjold and S. K. Ratkje, "Criteria for local equilibrium in a system with transport of heat and mass," Journal of Statistical Physics, vol. 78, no. 1-2, pp. 463-494, 1995.

[28] J. Xu, S. Kjelstrup, D. Bedeaux, and J. M. Simon, "Transport properties of $2 \mathrm{~F} \rightleftarrows \mathrm{F} 2$ in a temperature gradient as studied by molecular dynamics simulations," Physical Chemistry Chemical Physics, vol. 9, no. 8, pp. 969-981, 2007.

[29] J. Xu, S. Kjelstrup, and D. Bedeaux, "Molecular dynamics simulations of a chemical reaction; Conditions for local equilibrium in a temperature gradient," Physical Chemistry Chemical Physics, vol. 8, no. 17, pp. 2017-2027, 2006.

[30] A. Vergara, F. Capuano, L. Paduano, and R. Sartorio, "Lysozyme mutual diffusion in solutions crowded by poly(ethylene glycol)," Macromolecules, vol. 39, no. 13, pp. 4500-4506, 2006.

[31] T. Ikeda, M. Boero, and K. Terakura, "Hydration of alkali ions from first principles molecular dynamics revisited," Journal of Chemical Physics, vol. 126, no. 3, Article ID 034501, 9 pages, 2007.

[32] J. H. He, "Homotopy perturbation technique," Computer Methods in Applied Mechanics and Engineering, vol. 178, pp. 257-262, 1999.

[33] J. H. He, "Homotopy perturbation method: a new nonlinear analytical technique," Applied Mathematics and Computation, vol. 135, no. 1, pp. 73-79, 2003.

[34] J. H. He, "A simple perturbation approach to Blasius equation," Applied Mathematics and Computation, vol. 140, no. 2-3, pp. 217-222, 2003.

[35] P. D. Ariel, "Alternative approaches to construction of Homotopy perturbation Algorithms," Nonlinear Science Letters, vol. 1, pp. 43-52, 2010.

[36] Q. K. Ghori, M. Ahmed, and A. M. Siddiqui, "Application of homotopy perturbation method to squeezing flow of a newtonian fluid," International Journal of Nonlinear Sciences and Numerical Simulation, vol. 8, no. 2, pp. 179-184, 2007.

[37] T. Öziş and A. Yildirim, “A comparative study of He’s Homotopy Perturbation Method for determining frequency-amplitude relation of a nonlinear oscillator with discontinuities," International Journal of Nonlinear Sciences and Numerical Simulation, vol. 8, no. 2, pp. 243-248, 2007.
[38] S. J. Li and Y. X. Liu, "An improved approach to nonlinear dynamical system identification using PID neural networks," International Journal of Nonlinear Sciences and Numerical Simulation, vol. 7, no. 2, pp. 177-182, 2006.

[39] M. M. Mousa and S. F. Ragab, "Application of the homotopy perturbation method to linear and nonlinear schrödinger equations," Zeitschrift für Naturforschung, vol. 63, no. 3-4, pp. 140-144, 2008.

[40] V. Ananthaswamy and L. Rajendran, "Analytical Solution of Non-Linear Kinetic equation in a porous pellet," Global Journal of Pure and Applied Mathematics, vol. 8, pp. 101-111, 2012.

[41] S. Loghambal and L. Rajendran, "Mathematical modeling of diffusion and kinetics in amperometric immobilized enzyme electrodes," Electrochimica Acta, vol. 55, no. 18, pp. 5230-5238, 2010.

[42] A. Meena and L. Rajendran, "Mathematical modeling of amperometric and potentiometric biosensors and system of nonlinear equations-homotopy perturbation approach," Journal of Electroanalytical Chemistry, vol. 644, no. 1, pp. 50-59, 2010.

[43] A. Shanmugarajan, S. Alwarappan, S. Somasundaram, and R. Lakshmanan, "Analytical solution of amperometric enzymatic reactions based on Homotopy perturbation method," Electrochimica Acta, vol. 56, no. 9, pp. 3345-3352, 2011.

[44] V. Ananthaswamy and L. Rajendran, "Analytical solution of two-point non linear boundary value problems in a porous catalyst particles," International Journal of Mathematical Archieve, vol. 3, no. 3, pp. 810-821, 2012. 

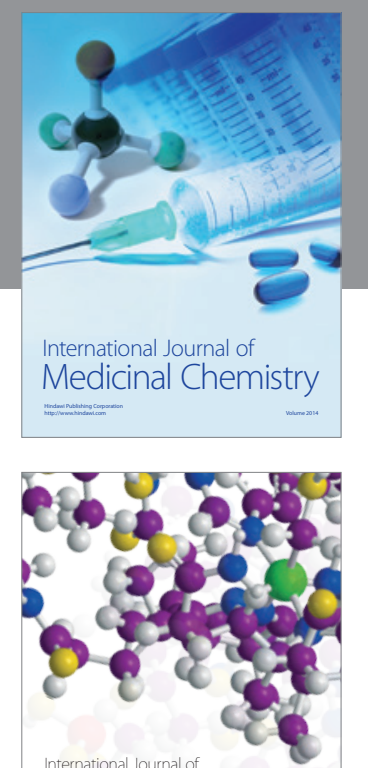

\section{Carbohydrate} Chemistry

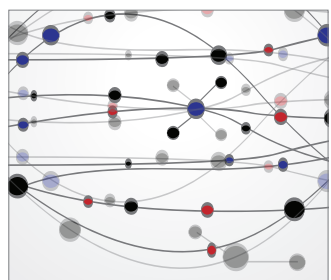

The Scientific World Journal
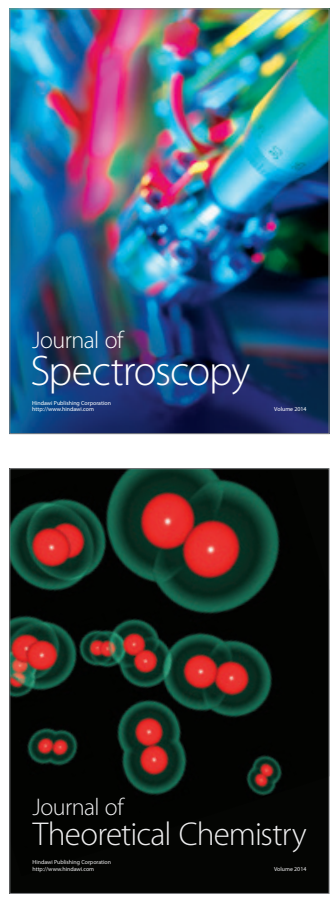
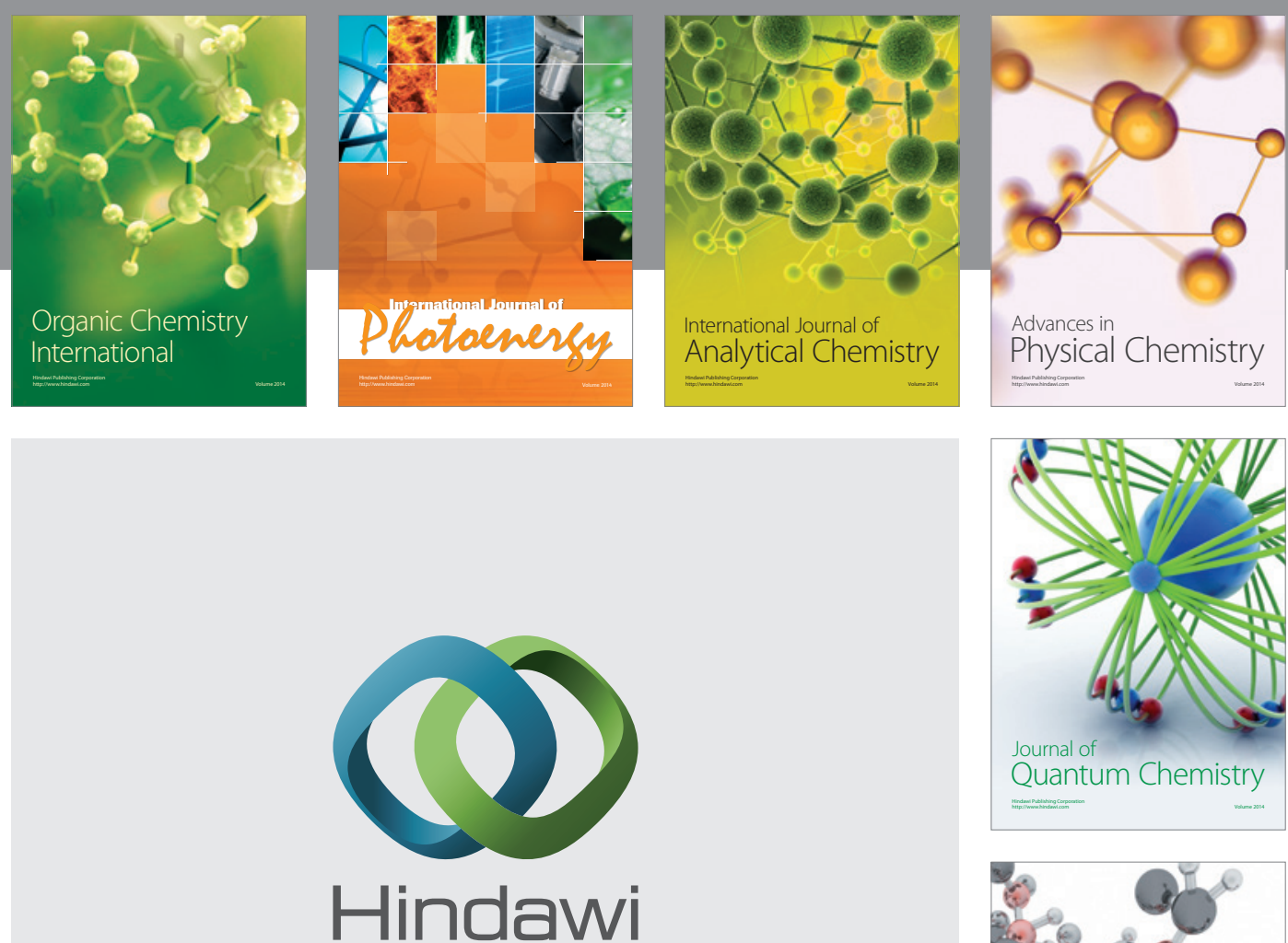

Submit your manuscripts at

http://www.hindawi.com

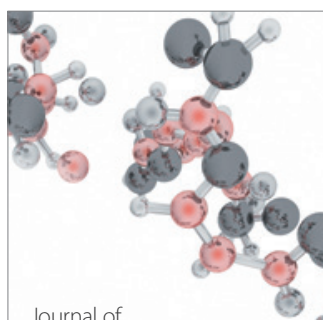

Analytical Methods

in Chemistry

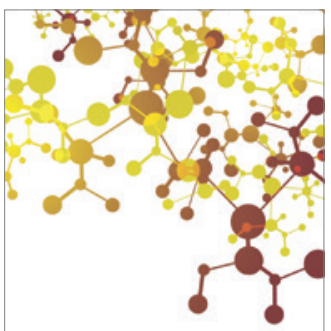

Journal of

Applied Chemistry

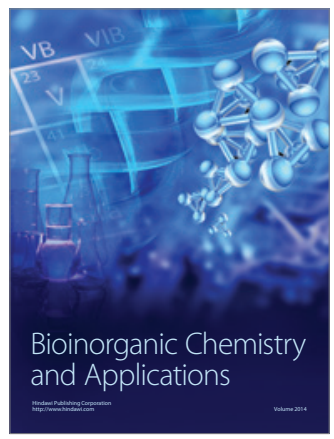

Inorganic Chemistry
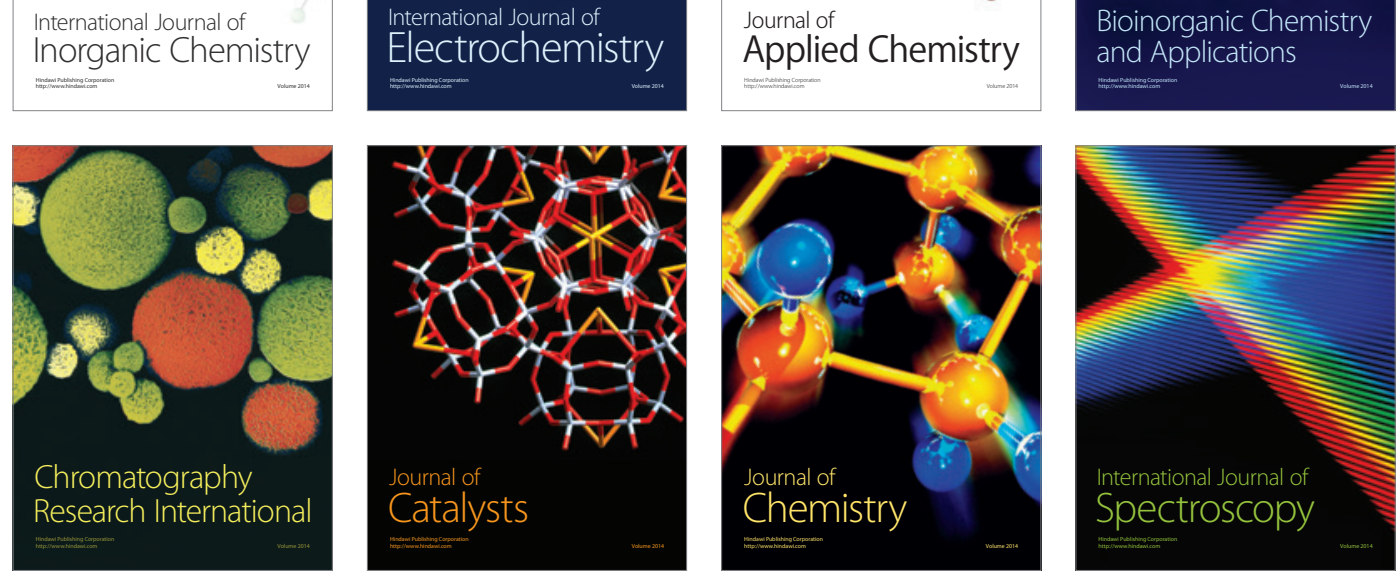